\title{
Synthesis of 4-(Arylmethyl)proline Derivatives
}

\author{
Simon Loosli ${ }^{\diamond}$ \\ Carlotta Foletti ${ }^{\triangleright}$ \\ Marcus Papmeyer \\ Helma Wennemers* (D) \\ ETH Zürich, Laboratory for Organic Chemistry, D-CHAB, \\ Vladimir-Prelog-Weg 3, 8093 Zürich, Switzerland \\ Helma.Wennemers@org.chem.ethz.ch \\ $\diamond$ These authors contributed equally. \\ Published as part of the 30 Years SYNLETT - Pearl Anniversary \\ Issue
}

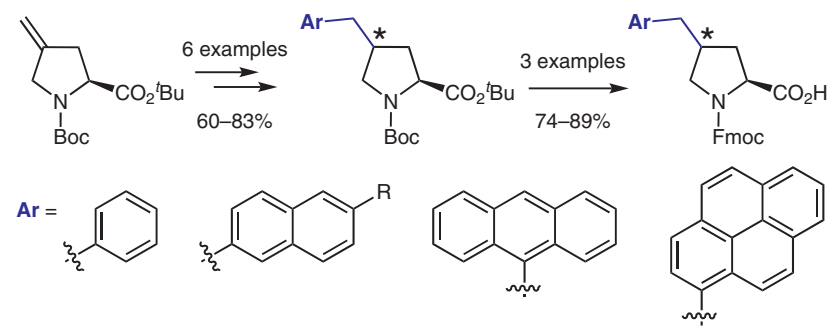

in transferring these reaction conditions, which rely on Wittig reactions of 4-oxoproline followed by hydrogenation, to larger aryl moieties (Scheme 1, top).

We therefore sought an alternative route and we envisioned Suzuki reactions between an organoborane-proline derivative and aryl halides as a strategy that might provide access to proline derivatives with various aryl groups (Scheme 1, bottom). Here, we report a general synthetic route to arylmethyl proline derivatives that permits the introduction of a broad range of aryl moieties at $C \gamma$.

Our synthetic route relies on the hydroboration of the Boc/t $\mathrm{Bu}$-protected 4-methyleneproline 5, which was obtained from $(2 S, 4 R)$-4-hydroxyproline $(\mathbf{1})$ by slight modification of a previously published procedure (Scheme 2). ${ }^{10}$ This four-step synthesis started with Boc-protection of $\mathbf{1}$, followed by oxidation to ketone 3 , protection of the carboxylic acid as the ${ }^{t} \mathrm{Bu}$ ester in $\mathbf{4}$, and introduction of an exocyclic methylene group by a Wittig reaction. ${ }^{11}$

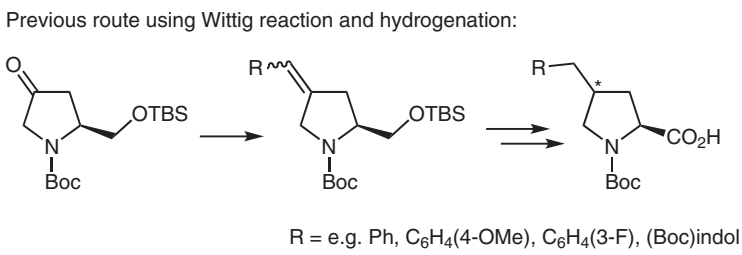

Herein, route using Suzuki reaction:

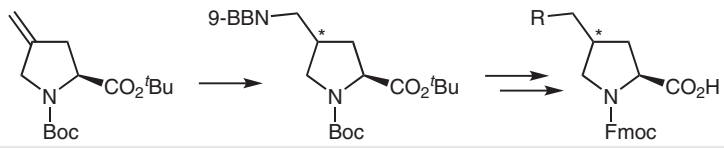

Scheme 1 Synthetic routes to 4-(arylmethyl)proline derivatives 
$\mathrm{HO}$
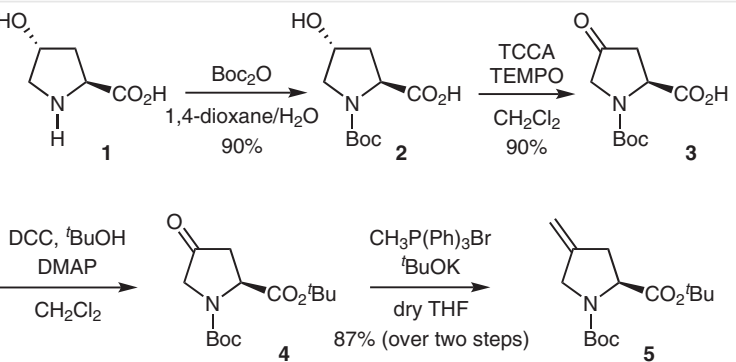

TCCA $=$ trichloroisocyanuric acid

Scheme 2 Synthesis of the common precursor tert-butyl N-(tertbutoxycarbonyl)-4-methyleneprolinate (5)

Hydroboration of the 4-methyleneproline 5 with 9-BBN provided the organoborane $\mathbf{6}$, which was used for the Suzuki reaction without further purification (Scheme 3, top). For the Suzuki reaction, various catalysts and conditions were explored by using 2-bromonaphthalene as a model aryl bromide. We focused in particular on catalysts that had proven valuable for cross-couplings with other amino acid derivatives (Scheme 3, bottom). ${ }^{12}$ Among the tested palladium-based catalysts, reactions with PEPPSI ${ }^{13}$ showed the highest conversion of $\mathbf{5}$ and 2-bromonaphthalene into the Suzuki reaction product $\mathbf{7 a}$. Under optimized conditions [5 $\mathrm{M}$ aq $\mathrm{KOH}, \operatorname{ArBr}$ (1.3 equiv), PEPPSI(3\% mol)], the 4-(2naphthylmethyl)proline derivative $7 \mathbf{a}$ was obtained in a yield of $83 \%$. Note that $3 \mathrm{~mol} \%$ of PEPPSI was enough to obtain these results. Because PEPPSI is more air-stable than other palladium catalysts, ${ }^{14}$ this catalyst was used for all further experiments.
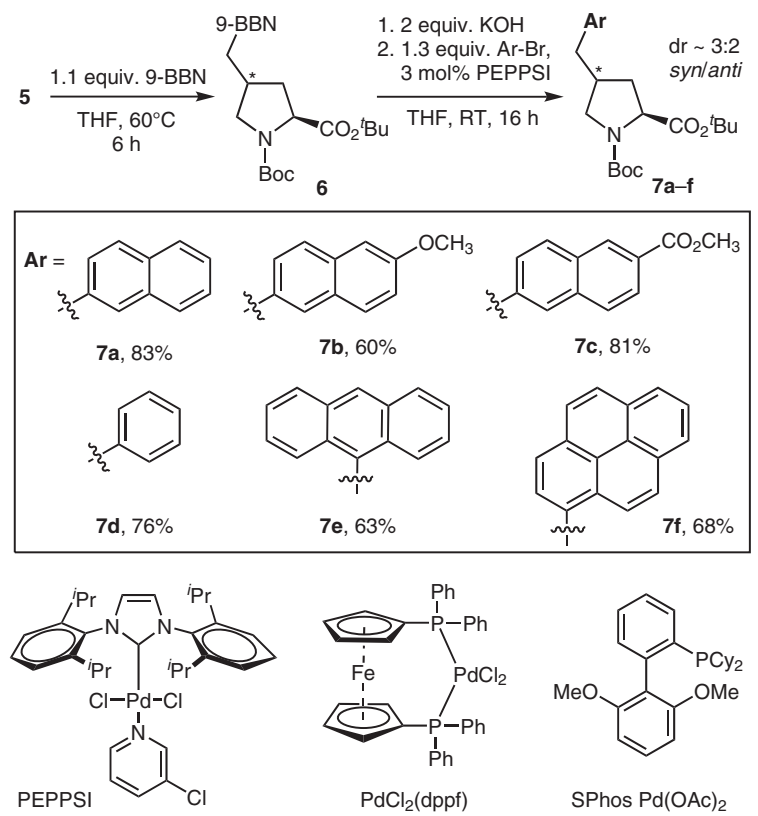

Scheme 3 Top: Suzuki cross-coupling reaction to yield various 4-(arylmethyl)proline derivatives 7a-f. Bottom: Catalysts tested in the Suzuki cross-coupling reaction.
Reassuringly, this route also permitted the synthesis of proline derivatives bearing substituted naphthyl moieties (7b and 7c) as well as phenyl (7d), 9-anthryl (7e), or pyren$1-y l$ (7f) substituents in good overall yields (60-83\%; Scheme 3). ${ }^{15}$ All derivatives were obtained with a diastereoselectivity of $\sim 3: 2$ in favor of the syn-product, as determined by analysis of ${ }^{1} \mathrm{H}$ NMR NOE spectroscopy. ${ }^{11}$

Because peptide syntheses typically require Fmoc-protected amino acids, we converted $\mathbf{7 a - c}$ into the respective Fmoc-amino acids 8a-c. Simultaneous removal of the ${ }^{t} \mathrm{Bu}$ protecting groups in $6 \mathrm{M} \mathrm{HCl}$ in 1,4-dioxane, and subsequent Fmoc-protection afforded $\mathbf{8 a - c}$ in yields of $74-89 \%$ (Scheme 4). The diastereoisomers were separated by preparative reverse-phase HPLC to obtain enantiomerically pure amino acids at a scale of up to $2.5 \mathrm{~g} \cdot{ }^{15,16}$

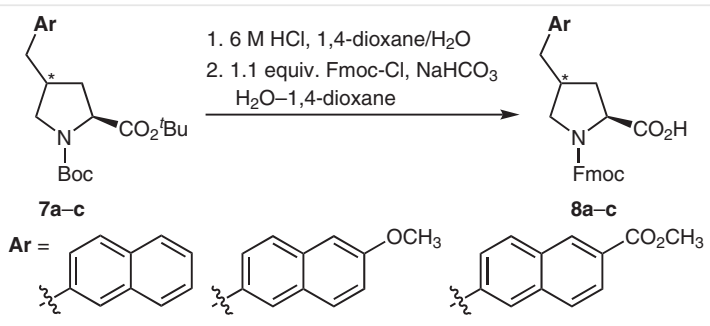

$8 \mathbf{8 a}, 85 \% \quad \mathbf{8 b}, 74 \% \quad 8 c, 89 \%$

Scheme 4 Synthesis of Fmoc-protected amino acids 8a-c

In conclusion, we have introduced a synthetic route to access proline derivatives bearing a variety of arylmethyl substituents at the $\gamma$-position. The products were obtained in good yields for every tested aromatic moiety. The diastereoselectivity of the hydroboration step was modest, but the diastereoisomeric products could be separated on a gram scale. Installation of a Fmoc-protecting group was straightforward. Thus, the route provides access to proline derivatives with a variety of arylmethyl moieties at $C \gamma$ that are suitably protected for solid-phase peptide synthesis. We envision these derivatives as being valuable additions to the toolkit of proline analogues for applications in chemistry and chemical biology.

\section{Funding Information}

The authors gratefully acknowledge financial support by the Swiss National Science Foundation (grant 200020_178805).

\section{Supporting Information}

Supporting information for this article is available online at https://doi.org/10.1055/s-0037-1611672.

\section{References and Notes}

(1) Shoulders, M. D.; Raines, R. T. Annu. Rev. Biochem. 2009, 78, 929.

(2) For examples, see: (a) Arnold, U.; Hinderaker, M. P.; Köditz, J.; Golbik, R.; Ulbrich-Hofmann, R.; Raines, R. T. J. Am. Chem. Soc. 2003, 125, 7500. (b) Lummis, S. C.; Beene, D. L.; Lee, L. W.; 
Lester, H. A.; Broadhurst, R. W.; Dougherty, D. A. Nature 2005, 438, 248. (c) Lieblich, S. A.; Fang, K. Y.; Cahn, J. K. B.; Rawson, J.; LeBon, J.; Ku, H. T.; Tirrell, D. A. J. Am. Chem. Soc. 2017, 139, 8384. (d) Metrano, A. J.; Abascal, N. C.; Mercado, B. Q.; Paulson, E. K.; Hurtley, A. E.; Miller, S. J. J. Am. Chem. Soc. 2017, 139, 492.

(3) Liu, J.; Wang, L. Synthesis 2017, 49, 960.

(4) Lewandowski, B.; Wennemers, H. Curr. Opin. Chem. Biol. 2014, $22,40$.

(5) (a) Remuzon, P. Tetrahedron 1996, 52, 13803. (b) Pandey, A. K.; Naduthambi, D.; Thomas, K. M.; Zondlo, N. J. J. Am. Chem. Soc. 2013, 135, 4333.

(6) (a) Del Valle, J. R.; Goodman, M. J. Org. Chem. 2003, 68, 3923. (b) Koskinen, A. M. P.; Helaja, J.; Kumpulainen, E. T. T.; Koivisto, J.; Mansikkamäki, H.; Rissanen, K. J. Org. Chem. 2005, 70, 6447.

(7) Foletti, C.; Kramer, R. A.; Mauser, H.; Jenal, U.; Bleicher, K. H.; Wennemers, H. Angew. Chem. Int. Ed. 2018, 57, 7729.

(8) (a) Ezquerra, J.; Pedregal, C.; Yruretagoyena, B.; Rubio, A.; Carreño, M. C.; Escribano, A.; García Ruano, J. L. J. Org. Chem. 1995, 60, 2925. (b) Rawson, D. J.; Brugier, D.; Harrison, A.; Hough, J.; Newman, J.; Otterburn, J.; Maw, G. N.; Price, J.; Thompson, L. R.; Turnpenny, P.; Warren, A. N. Bioorg. Med. Chem. Lett. 2011, 21, 3771.

(9) Krapcho, J.; Turk, C.; Cushman, D. W.; Powell, J. R.; DeForrest, J. M.; Spitzmiller, E. R.; Karanewsky, D. S.; Duggan, M.; Rovnyak, G.; Schwartz, J.; Natarajan, S.; Godfrey, J. D.; Ryono, D. E.; Neubeck, R.; Atwal, K. S.; Petrillo, E. W. Jr.J. Med. Chem. 1988, 31, 1148.

(10) (a) Herdewijn, P.; Claes, P. J.; Vanderhaeghe, H. Can. J. Chem. 1982, 60, 2903. (b) De Luca, L.; Giacomelli, G.; Porcheddu, A. Org. Lett. 2001, 3, 3041.

(11) For details, see the Supporting Information.

(12) For the use of the Suzuki reaction to prepare amino acids, see: (a) Campbell, A. D.; Raynham, T. M.; Taylor, R. J. K. Tetrahedron Lett. 1999, 40, 5263. (b) Sabat, M.; Johnson, C. R. Org. Lett. 2000 , 2, 1089. (c) Lu, X.; Xiao, B.; Shang, R.; Liu, L. Chin. Chem. Lett. 2016, 27, 305.

(13) O’Brien, C. J.; Kantchev, E. A. B.; Valente, C.; Hadei, N.; Chass, G. A.; Lough, A.; Hopkinson, A. C.; Organ, M. G. Chem. Eur. J. 2006, $12,4743$.

(14) Ray, L.; Shaikh, M. M.; Ghosh, P. Dalton Trans 2007, 4546.

(15) tert-Butyl (4S/4R)-N-(tert-Butoxycarbonyl)-4-(2-naphthylmethyl)-L-prolinate (7a); Typical Procedure

An oven-dried Schlenk flask was charged with methylene derivative 5 ( $4.0 \mathrm{~g}, 14.1 \mathrm{mmol}, 1$ equiv) under $\mathrm{N}_{2}$. A $0.5 \mathrm{M}$ soln of 9BBN in THF (31.0 mL, $15.5 \mathrm{mmol}, 1.1$ equiv) was added in one portion, and the solution was stirred vigorously at $60^{\circ} \mathrm{C}$ for $6 \mathrm{~h}$. The mixture was then allowed to cool to r.t. and $5 \mathrm{M}$ aq. $\mathrm{KOH}$ ( $5.6 \mathrm{~mL}, 5 \mathrm{M}, 28.0 \mathrm{mmol}$, 2 equiv) was added. The mixture was stirred for $20 \mathrm{~min}$, then 2-bromonaphthalene (7a; $3.8 \mathrm{~g}, 18.36$ mmol, 1.3 equiv) was added together with PEPPSI $(287.7 \mathrm{mg}$, $423 \mu \mathrm{mol}, 0.03$ equiv). The mixture was stirred for a further $16 \mathrm{~h}$ at r.t., then $\mathrm{H}_{2} \mathrm{O}(120 \mathrm{~mL})$ and EtOAc $(120 \mathrm{~mL})$ were added and the phases were separated. The aqueous phase was extracted with EtOAc $(3 \times 120 \mathrm{~mL})$, and the organic layers were combined, washed with brine, dried $\left(\mathrm{MgSO}_{4}\right)$, and concentrated. The resulting yellow-brown oil (9.9 $\mathrm{g}$ ) was purified by column chromatography (silica gel, 0-25\% EtOAc-hexane) to give a colorless oil; yield: $4.8 \mathrm{~g}$ (83\%).

${ }^{1} \mathrm{H}$ NMR $\left(500 \mathrm{MHz}, \mathrm{C}_{2} \mathrm{Cl}_{4} \mathrm{D}_{2}, 60{ }^{\circ} \mathrm{C}\right): \delta=7.82-7.71(\mathrm{~m}, 3 \mathrm{H}), 7.59-$ $7.52(\mathrm{~m}, 1 \mathrm{H}), 7.48-7.37(\mathrm{~m}, 2 \mathrm{H}), 7.27(\mathrm{dd}, J=8.4,1.7 \mathrm{~Hz}, 1 \mathrm{H})$, 4.26-4.02 (m, $1 \mathrm{H}), 3.75-3.55(\mathrm{~m}, 1 \mathrm{H}), 3.14(\mathrm{dd}, J=10.6,9.0 \mathrm{~Hz}$, $1 \mathrm{H}), 2.89-2.76(\mathrm{~m}, 2 \mathrm{H}), 2.72-2.44(\mathrm{~m}, 1 \mathrm{H}), 2.42-1.88(\mathrm{~m}, 1 \mathrm{H})$, 1.63 (ddd, $J=12.8,9.5,7.9 \mathrm{~Hz}, 1 \mathrm{H}$ ), $1.51-1.33$ (m, $18 \mathrm{H}) .{ }^{13} \mathrm{C}$
NMR (126 MHz, $\left.\mathrm{C}_{2} \mathrm{Cl}_{4} \mathrm{D}_{2}, 60^{\circ} \mathrm{C}\right): \delta=172.2,172.0,153.6,137.6$, $137.4,133.5,133.5,132.1,128.1,128.1,127.6,127.5,127.4$, $127.2,127.1,126.8,126.8,126.1,125.4,80.9,80.8,79.6,79.5$, 59.8, 59.7, 52.1, 51.6, 39.3, 39.2, 37.7, 36.7, 36.4, 28.4, 28.0, 28.0. HRMS (ESI+): $m / z[\mathrm{M}+\mathrm{H}]^{+}$calcd $\mathrm{C}_{25} \mathrm{H}_{34} \mathrm{NO}_{4}: 412.2482$; found: 412.2485 .

(4S)- and (4R)-1-[(9H-Fluoren-9-ylmethoxy)carbonyl]-4-(2naphthylmethyl)-L-proline (8a); Typical Procedure

Prolinate 7a (4.8 g, $11.7 \mathrm{mmol}, 1$ equiv) was dissolved in a $6 \mathrm{M}$ soln of $\mathrm{HCl}$ in 1,4-dioxane $(110 \mathrm{~mL})$, and the mixture was stirred for $3 \mathrm{~h}$ at r.t. The pH was adjusted to 8-9 with sat. aq $\mathrm{NaHCO}_{3}$, then a soln of $\mathrm{FmocCl}$ ( $3.6 \mathrm{~g}, 14.0 \mathrm{mmol}, 1.2$ equiv) in 1,4-dioxane $(50 \mathrm{~mL})$ was added, and the mixture was stirred at r.t. for $2 \mathrm{~h}$. Low-boiling volatiles were removed under reduced pressure, and EtOAc $(50 \mathrm{~mL})$ was added. The solution was acidified to $\mathrm{pH} \mathrm{2-3}$ with $1 \mathrm{M} \mathrm{HCl}$, and the organic phase was separated and extracted with EtOAc $(3 \times 100 \mathrm{~mL})$. The combined organic layers were washed with brine, dried $\left(\mathrm{MgSO}_{4}\right)$, and filtered. All volatiles were removed under reduced pressure, and the product was purified by column chromatography (silica gel, $0-5 \% \mathrm{MeOH}$ in $\mathrm{CH}_{2} \mathrm{Cl}_{2}$ with $0.1 \% \mathrm{HCO}_{2} \mathrm{H}$ ) to give a white powder: yield: $4.7 \mathrm{~g}(84 \%)$. The diastereoisomers were subsequently separated by reverse-phase semipreparative HPLC [Reprosil-Gold $120 \mathrm{C} 18,10 \mu \mathrm{m} ; 250 \times 30 \mathrm{~mm}$ column, MeCN and $\left.\mathrm{H}_{2} \mathrm{O}-\mathrm{MeCN}-\mathrm{TFA}(100: 1: 0.1)\right]$.

(4S)-Diastereomer

$[\alpha]_{D}-40.7 \pm 0.5$ (c 0.2, MeOH). TLC (silica gel, $2 \% \mathrm{MeOH}$ in $\mathrm{CH}_{2}-$ $\mathrm{Cl}_{2}$ ): $R_{f}=0.56$. FTIR (neat): 3051, 2923, 1701, 1421, 1352, 1247, $1176,1122,1006,972,843,739 \mathrm{~cm}^{-1}$.

${ }^{1} \mathrm{H}$ NMR $\left(500 \mathrm{MHz}, \mathrm{C}_{2} \mathrm{Cl}_{4} \mathrm{D}_{2}, 60{ }^{\circ} \mathrm{C}\right): \delta=7.92-7.79(\mathrm{~m}, 3 \mathrm{H}$; $\mathrm{Ar})$, 7.77-7.60 (m, $3 \mathrm{H}$; Ar), 7.59-7.44 (m, $4 \mathrm{H} ; \mathrm{Ar}), 7.43-7.21(\mathrm{~m}, 5$ $\mathrm{H}$; Ar), 4.55-4.41 (m, $\left.2 \mathrm{H} ; \mathrm{CH}_{2}-\mathrm{Fmoc}\right), 4.41-4.28(\mathrm{~m}, 1 \mathrm{H} ; \mathrm{H \alpha})$,

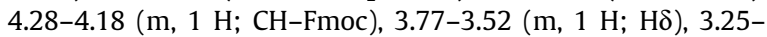
$3.16(\mathrm{~m}, 1 \mathrm{H} ; \mathrm{H} \delta), 3.01-2.79\left(\mathrm{~m}, 2 \mathrm{H} ; \mathrm{CH}_{2}-\mathrm{Naph}\right), 2.57$ (hept, $J=7.7 \mathrm{~Hz}, 1 \mathrm{H} ; \mathrm{H} \gamma), 2.50-2.36(\mathrm{~m}, 1 \mathrm{H} ; \mathrm{H} \beta), 2.12-1.93(\mathrm{~m}, 1 \mathrm{H}$; $\mathrm{H} \beta) .{ }^{13} \mathrm{C}$ NMR $\left(500 \mathrm{MHz}, \mathrm{C}_{2} \mathrm{Cl}_{4} \mathrm{D}_{2}, 60{ }^{\circ} \mathrm{C}\right): \delta=173.2\left(\mathrm{CO}_{2} \mathrm{H}\right), 156.4$ $\left(\mathrm{C}=\mathrm{O}_{\text {Fmoc }}\right), 143.5(\mathrm{Ar}), 141.1(\mathrm{Ar}), 137.0(\mathrm{Ar}), 133.4(\mathrm{Ar}), 132.1$ (Ar), 128.2 (Ar), 127.6 (Ar), 127.5 (Ar), 127.4 (Ar), 127.0 (Ar), 126.9 (Ar), 126.7 (Ar), 126.1 (Ar), $125.5(\mathrm{Ar}), 124.8(\mathrm{Ar}), 119.8$ (Ar), $67.9\left(\mathrm{CH}_{2}-\mathrm{Fmoc}\right), 59.4(\mathrm{C} \alpha), 52.2(\mathrm{C} \delta), 47.1$ (CH-Fmoc), $39.7(\mathrm{C} \gamma), 38.8\left(\mathrm{CH}_{2}-\mathrm{Naph}\right), 34.4(\mathrm{C} \beta)$. HRMS (ESI+): $\mathrm{m} / \mathrm{z}[\mathrm{M}+$ $\mathrm{H}]^{+}$calcd for $\mathrm{C}_{31} \mathrm{H}_{28} \mathrm{NO}_{4}$ : 478.2013; found: 478.2003.

\section{(4R)-Diastereomer}

$[\alpha]_{D}-10.8 \pm 0.3\left(c 0.2, \mathrm{MeOH}\right.$ ). TLC (silica gel, $2 \% \mathrm{MeOH}$ in $\mathrm{CH}_{2}-$ $\mathrm{Cl}_{2}$ ): $R_{f}=0.56$. FTIR (neat): $3045,2966,1700,1661,1417,1351$, $1241,1282,1122,1002,947,887,737 \mathrm{~cm}^{-1}$.

${ }^{1} \mathrm{H}$ NMR $\left(500 \mathrm{MHz}, \mathrm{C}_{2} \mathrm{Cl}_{4} \mathrm{D}_{2}, 60{ }^{\circ} \mathrm{C}\right): \delta=7.91-7.80(\mathrm{~m}, 3 \mathrm{H} ; \mathrm{Ar})$, 7.73 (dd, $J=7.6,2.9 \mathrm{~Hz}, 2 \mathrm{H} ; \mathrm{Ar}), 7.62(\mathrm{~s}, 1 \mathrm{H} ; \mathrm{Ar}), 7.59-7.48$ (m, $4 \mathrm{H} ; \mathrm{Ar}), 7.39$ (tt, $J=7.6,1.4 \mathrm{~Hz}, 2 \mathrm{H} ; \mathrm{Ar}), 7.35-7.27$ (m, $3 \mathrm{H} ; \mathrm{Ar})$, 4.56-4.36 (m, $3 \mathrm{H}$; Ha, $\left.\mathrm{CH}_{2}-\mathrm{Fmoc}\right), 4.32-4.19(\mathrm{~m}, 1 \mathrm{H}$; $\mathrm{CH}-$

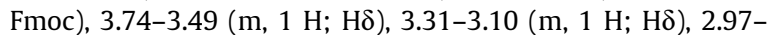
2.80 (m, $2 \mathrm{H} ; \mathrm{CH}_{2}-\mathrm{Naph}$ ), 2.80-2.65 (m, $\left.1 \mathrm{H} ; \mathrm{H} \gamma\right), 2.47-1.88$ (m, $2 \mathrm{H} ; \mathrm{HB}) .{ }^{13} \mathrm{C}$ NMR $\left(500 \mathrm{MHz}, \mathrm{C}_{2} \mathrm{Cl}_{4} \mathrm{D}_{2}, 60{ }^{\circ} \mathrm{C}\right): \delta=173.8\left(\mathrm{CO}_{2} \mathrm{H}\right)$, $156.1\left(\mathrm{C}=\mathrm{O}_{\mathrm{Fmoc}}\right), 143.5(\mathrm{Ar}), 141.1(\mathrm{Ar}), 136.8(\mathrm{Ar}), 133.4(\mathrm{Ar})$, $132.1(\mathrm{Ar}), 128.2(\mathrm{Ar}), 127.6(\mathrm{Ar}), 127.5(\mathrm{Ar}), 127.4(\mathrm{Ar}), 127.0$ (Ar), 127.0 (Ar), 126.8 (Ar), 126.1 (Ar), 125.5 (Ar), 124.8 (Ar), 119.82 (Ar), $67.9\left(\mathrm{CH}_{2}-\mathrm{Fmoc}\right), 59.2(\mathrm{C} \alpha), 51.7(\mathrm{C}), 47.1(\mathrm{CH}-$ Fmoc), $38.9\left(\mathrm{CH}_{2}-\mathrm{Naph}, \mathrm{C} \gamma\right), 34.3(\mathrm{C} \beta)$. HRMS (ESI+): $\mathrm{m} / z$ [M + $\mathrm{H}]^{+}$calcd $\mathrm{C}_{31} \mathrm{H}_{28} \mathrm{NO}_{4}$ : 478.2013; found: 478.2003.

(16) Note that the Suzuki reaction is not compatible with the use of Fmoc-protected amines. The stereochemistry at the stereogenic centers was retained during the synthesis. 